\title{
Prácticas educativas familiares y mediación parental vs dispositivos móviles
}

\author{
Antonia Ramírez García y Rocío Gómez Moreno
}

Universidad de Córdoba- España

\section{RESUMEN}

Las prácticas educativas familiares han cambiado fruto de una sociedad digitalizada, especialmente las estrategias de mediación aplicadas por los progenitores respecto al uso y consumo de dispositivos móviles en el hogar. La investigación de carácter cuantitativo utilizó como instrumentos de recogida de información los cuestionarios s EMBU-C y EU Kids online II, aplicándose a 180 escolares de $5^{\circ}$ y $6^{\circ}$ curso de Educación Primaria. Los resultados evidencian una práctica familiar basada en la calidez emocional y en la protección del menor, así como una implementación mayoritaria de estrategias de mediación activas en el uso y consumo de dispositivos móviles. En conclusión, es necesario sistematizar las distintas clasificaciones existentes sobre mediación parental, no solo para identificar cuáles son las más empleadas, sino también para determinar las más eficaces en relación a la educación mediática en el hogar.

Palabras clave: internet, mediación parental, prácticas parentales, dispositivos móviles, educación mediática.

\section{Family educational practices and parental mediation vs mobile and internet devices}

\begin{abstract}
Family educational practices have changed as a result of a digitized society, especially the mediation strategies applied by parents regarding the use and consumption of mobile devices at home. The quantitative research used as information collection instruments the EMBU-C and EU Kids online II questionnaires, applied to 180 school children in 5th and 6th year of Primary Education. Results show a family practice based on emotional warmth and child protection, as well as a majority implementation of active mediation strategies in the use and consumption of mobile devices. In conclusion, it is necessary to systematize the different existing classifications on parental mediation, not only to identify which are the most used, but also to determine the most effective ones in relation to media literacy at home.
\end{abstract}

Keywords: Internet, parental mediation, parental practices, mobile devices, media literacy. 


\section{Introducción}

\subsection{Los estilos parentales}

$\mathrm{Al}$ analizar los orígenes de los rasgos y características personales de los menores, resulta inevitable pensar en la familia. Desde la teoría de sistemas se constata que estos están condicionados por el entorno e influencian a otros subsistemas desde una perspectiva holística. Al tiempo, los progenitores son considerados uno de los agentes de socialización que ejerce mayor influencia en sus descendientes (Wilfred y Allan, 2016). Por tanto, la familia es la primera esfera de organización social en la vida de una persona (Martínez-Vasallo, 2015).

La familia ha evolucionado a la par que el resto de la sociedad en cuanto a nuevos modelos familiares -nuclear, monoparental, familia extensa, reconstituida, etc.- (Cuero, Gutiérrez e Infante, 2015; Deslandes, 2019) o funciones que desempeñan, destacando entre ellas la socialización.

Estas funciones se desarrollan condicionadas por el estilo parental de las familias. A mediados del siglo pasado surgió el concepto de estilo parental, acuñado por Baumrind (1966) y entendido como la forma de actuar de los progenitores respecto a sus hijos ante situaciones cotidianas de la vida, toma de decisiones o resolución de conflictos, comunicación y tiempo de convivencia que se dedican, así como promoción del éxito.

Se han encontrado distintos términos como sinónimos de este concepto, "estilo de crianza", "estilo educativo" o "prácticas educativas familiares" (Capano, Tornaría, Del Luján y Massonier, 2016; Jorge y González, 2017).

Autores como Baumrind (1991), Ceballos y Rodrigo (1998) o García, Pelegrina y Lendínez (2002) centraron sus estudios en la caracterización de distintos estilos parentales mediante la combinación de dos dimensiones: afecto y comunicación y control y disciplina. A partir de ello se establecieron los estilos parentales iniciales (autoritario, permisivo y democrático) y una ampliación posterior -autoritario, democrático, permisivo e indiferente-.

Los estilos de crianza de los progenitores actúan de moduladores de sus hijos (Cabrera y Salazar, 2014). Un estilo parental autoritario provoca denigración, indiferencia, rechazo y abandono de los padres hacia sus hijos (Claesson y Sohlberg 2002; Han y Kim 2012). Por otro lado, Chorot, Valiente, Magaz, Santed y Sandin (2017), evidencian que los comportamientos parentales de sobreprotección predicen síntomas de trastornos de ansiedad. Sin embargo, los comportamientos parentales de calidez emocional se relacionan con bajos niveles de síntomas depresivos.

Recientemente, la clasificación de estilos de crianza ha sido modificada y ampliada ante la necesidad de incorporar prácticas parentales vinculadas al uso de Internet (Valcke, Bonte, Wever y Rots, 2010), lo que demuestra los efectos que la sociedad líquida produce en distintos ámbitos, el familiar entre ellos.

\subsection{La e-society}

La teoría de sistemas sitúa al menor en otro contexto de influencia. La sociedad, o mejor dicho la e-society, incide directamente en los hogares a través de la presencia de una equipación tecnológica y de su conexión a Internet.

La última encuesta del Instituto Nacional de Estadística (2019) sobre el Equipamiento y Uso de Tecnologías de la Información y Comunicación en los hogares españoles muestra un aumento de 5 puntos en la tasa de hogares con conexión a Internet respecto al año anterior, llegando a alcanzar un 91.4\%. El 99.1\% dispone de televisión, el 80.9\% cuenta con ordenador (de cualquier tipo, incluidos netbooks, tablets, portátiles, etc.), el 74.9\% posee tele- fonía fija y el 98.5\% telefonía móvil. Con porcentajes inferiores, los hogares españoles han incorporado distintos dispositivos: cadena musical $(45.3 \%)$, radio $(67.8 \%)$, MP3 o MP4 $(36.9 \%)$, vídeo (24.8\%), DVD (49.8\%), e-book (25\%) y tablet (56.8\%).

En la misma línea, el informe de la Sociedad Digital en España publicado por la Fundación Telefónica (2019, p.12) expresa que el Smartphone "sigue ganando peso como dispositivo mayoritario", que se produce un incremento de la mensajería instantánea, que la privacidad y la seguridad son las mayores preocupaciones para los internautas (situación que también corrobora el ONTSI, 2019) y que el mayor consumo de contenidos multimedia tiene lugar a través de las plataformas y del Smartphone. Estas tendencias son también reveladas en el Key Takeaways from Mary Meeker's 2019 Internet Trends Report, que muestra el crecimiento continuado de los usuarios de Internet, el aumento considerable del tiempo diario de consumo digital (especialmente vídeos) y la supremacía del teléfono móvil sobre otros dispositivos electrónicos.

\subsection{La mediación parental}

Las familias tienen que afrontar esta nueva realidad social que afecta directamente a su forma de vida y al estilo de crianza (Duggan, Lenhart, Lampe y Ellison, 2015; Ramírez-García, Salcines-Talledo y González-Fernández, 2020; Rodríguez-Ruiz; Álvarez-Blanco, Martínez-González y Epstein, 2019; Torrecillas-Lacave, Vázquez-Barrio, Suárez y Fernández-Martínez, 2020; Wartella, Rideout, Lauricella y Connell, 2013), , para minimizar o reconducir el impacto de los nuevos dispositivos móviles en el hogar. Esto requiere nuevas estrategias que los progenitores han de utilizar en los contextos actuales de relación digital con sus hijos.

Como ocurría con la televisión, la mediación puede ser una estrategia para afrontar el desafío tecnológico (Garmendia, Casado, Martínez y Garitaonandia, 2013; Valcke, De Weber, Van Keer y Schellens, 2011). La mediación parental en un contexto digital se entiende como las diversas actividades parentales que permiten gestionar y regular las acciones de sus hijos con los dispositivos móviles (Livingstone, Mascheroni, Dreier, Chaudron y Lagae, 2015)

Existen diferentes clasificaciones sobre tipologías de mediación parental. La más tradicional distingue entre mediación restrictiva, activa y uso compartido (Gentile, Nathanson, Rasmussen, Reimer y Walsh, 2012; Nikken y Jansz, 2006). Esta clasificación se ha convertido en una seña de identidad generalizada en las familias de culturas occidentales en relación a los niños y los medios de comunicación (Zaman y Mifsud, 2017).

No obstante, resulta necesario redefinir esta categorización, pues los medios de comunicación han evolucionado y poseen características distintas (conectividad, individualidad, tamaño, etc.), lo que posibilita el desarrollo de otros modelos (Brito, Francisco, Dias y Chandron, 2017; Daneels y Vanwynsberghe, 2017; Lauricella, Wartella y Rideout, 2015).

Para sistematizar estrategias de mediación parental respecto al uso de Internet, Symons, Ponnet, Emmery, Walrave y Heizman (2017) proponen: restricciones de interacción, monitoreo, restricción de acceso, supervisión y uso compartido, mediación técnica y mediación interpretativa. En España, la investigación de Bartau-Rojas, Aierbe-Barandiaran y Oregui-González (2018) con niños de Educación Primaria sobre Internet reveló siete modalidades de mediación parental: establecimiento y aplicación de normas, organización espacio-temporal, supervisión, comunicación, enseñanza, modelado y estimulación.

A pesar de esta abundancia de clasificaciones, su empleo $\mathrm{y}$ eficacia resulta muy desigual en diferentes investigaciones 
(Bartau-Rojas, Aierbe-Barandiaran y Oregui-González, 2018), al tiempo que no existen resultados concluyentes de aquellas estrategias más efectivas (Marciales y Cabra, 2011), pudiéndose diferenciar tres grandes grupos de investigaciones:

1) Las que evidencian un uso de estrategias de carácter activo por las familias: Aierbe, Orozco y Medrano (2014), Giménez, Luengo y Bartrina (2017), Garmendia, Casado, Martínez y Garitaonandia (2013), entre otros.

2) Las que muestran un empleo de estrategias restrictivas: Fletcher y Blair (2014) o Monks, Mahdavi y Rix (2016).

3) Las que recogen un uso indistintos de estrategias por parte de las familias: Helsper, Kalmus, Hasebrink, Sagvari y de Haan (2013) o Martínez de Morentin y Medrano (2012).

\section{Objetivo de la investigación}

El objetivo de esta investigación es determinar qué variables inciden en las prácticas educativas familiares en relación a la gestión parental de dispositivos electrónicos, Internet y la aplicación de estrategias de mediación. De manera específica, se establecen los siguientes objetivos: 1) Identificar las prácticas educativas parentales más utilizadas por las familias cordobesas en relación al uso y consumo de dispositivos móviles de sus hijos; 2) Determinar qué tipo de mediación parental es la más empleada por las familias cordobesas en relación al uso y consumo de dispositivos móviles en el hogar.

\section{Método}

\subsection{Participantes}

Los participantes fueron 180 escolares cordobeses, un $45.6 \%$ eran chicas y un $54 \%$ chicos. Sus edades estaban comprendidas entre los 11 y los 13 años, siendo la edad media de 11.32 años. Las familias son nucleares $(87.6 \%)$, reconstituidas $(6.6 \%)$, monoparentales $(2.5 \%)$, extensas $(1.7 \%)$, adoptivas $(0.8 \%) \mathrm{u}$ otro tipo familiar $(0.8 \%)$.

En cuanto a la profesión materna, un $28.4 \%$ ocupa profesiones liberales, un $20.7 \%$ realiza tareas domésticas en su propio hogar, otro $12.4 \%$ tiene empleos administrativos, un $8.9 \%$ dirigen pequeñas empresas o son empleadas de la administración pública, un $8.3 \%$ se dedica a un trabajo sin cualificación específica, el $5.3 \%$ de ellas son pequeñas empresarias, un $3.6 \%$ posee un trabajo cualificado en construcción, industrias manufactureras o artesanía, un $2.4 \%$ son desempleadas y, por último, un $1.2 \%$ trabajan en servicios de protección.

El 33.1\% de los padres ejercen una profesión liberal, el 17.2\% al trabajo cualificado en construcción, industrias manufactureras o artesanía (mayoritariamente en el sector de la joyería), el 11.7\% trabaja en el sector servicios, el 9.2\% se dedica a la dirección de empresas o administración pública, el $8.6 \%$ son pequeños empresarios, un $8 \%$ de ellos son empleados de tipo administrativo o técnico de apoyo, el 5.5\% son trabajadores sin cualificación específica, un $4.3 \%$ se dedican a servicios de protección y seguridad $\mathrm{y}$, finalmente, un $1.2 \%$ son desempleados y/o trabajadores en las tareas domésticas de su propia casa.

Los dispositivos con los que los menores acceden a Internet son: dispositivos móviles como tablets $(76.3 \%)$, televisión $(64.6 \%)$, videoconsola $(57.5 \%)$, teléfono móvil $(51.7 \%)$, ordenador de sobremesa compartido (45.9\%), portátil compartido $(30.1 \%)$, propio portátil $(27.8 \%)$ y propio ordenador de sobremesa $(19.4 \%)$.

\section{Variables e instrumentos}

En este estudio se recabó información sobre las siguientes variables sociodemográficas: sexo, edad, estructura familiar, profesión de la madre, profesión del padre y dispositivos de acceso a Internet.

En cuanto a las variables que se analizaron fueron por un lado, la calidez emocional, el rechazo y la sobreprotección, pertenecientes al cuestionario $s E M B U-C$. Esta es una versión corta del cuestionario original Egna Minnen avBarndoms Uppfostran (EMBU), adaptada a niños y desarrollada por Arrindell et al. (1999) que está compuesta por 23 ítems y tres subescalas (1 a 4 puntos) que miden los estilos educativos parentales en relación a la calidez emocional (6 ítems), rechazo (7 ítems) y sobreprotección (9 ítems). Esta versión corta del s EMBU está recomendada para su uso en diversos países como equivalente funcional del EMBU original (Liu et al., 2018).

La calidez emocional es definida por Penelo, Viladrich y Doménech (2009) como aquellos elementos físicos y verbales (besos, abrazos y cumplidos) de aceptación y estimulación por parte de los padres, lo que implica confianza, apoyo sin intrusión y tener en cuenta las opiniones del menor. Por su parte, el rechazo, a juicio de estos mismos autores, se identifica con la hostilidad de los progenitores tanto física como verbal, conductas abusivas y punitivas, degradación, menosprecio, agresión, crítica, avergonzamiento con burlas, indiferencia hacia las opiniones, negligencia e, incluso, rechazo en favor de otros hermanos. Igualmente, la sobreprotección la describen como el intento de los padres por regular y controlar la conducta de su hijo o hija, con una preocupación excesiva, imposición y obediencia a normas estrictas, altas expectativas de logro y chantaje emocional.

Por otro lado, se contemplaron las variables percepción sobre el conocimiento parental de las acciones que realiza el menor en Internet, interés parental en dichas acciones, acciones parentales, limitación parental, tener en cuenta opinión parental, nuevas acciones parentales y mediación familiar (mediación activa del uso de Internet o co-uso; mediación restrictiva; mediación restrictiva de seguridad en Internet; monitorización y, finalmente, mediación técnica). Estas variables forman parte de un cuestionario, el EU kids online II, desarrollado por Livingstone y Haddon (2009) con el objetivo de analizar los comportamientos de los niños y niñas en relación al uso de los dispositivos móviles e Internet.

Para Dürager y Sonck (2014) la mediación activa o instructiva implica la explicación y la discusión sobre el contenido digital al que acceden los menores en Internet, mientras que la mediación restrictiva supone la fijación de reglas sobre dónde, cuándo, por cuánto tiempo y a qué se debe acceder en Internet; por su parte, la mediación denominada co-using (co-uso) incluye todas las actividades que se llevan a cabo con los medios de manera compartida entre padres e hijos (Nikken y Jansz, 2006). Estos tres tipos de mediación han sido complementados con otras dos categorías debido, básicamente, a las características propias del uso de Internet. Así, se denomina monitorización a la mediación que se centra en controlar o revisar las actividades que realizan los menores en Internet y la mediación técnica, que consiste en la restricción del contenido en línea o el tiempo de permanencia en la Red aplicando prohibiciones o filtros técnicos (Livingstone y Helsper, 2008; Sonck et al., 2013).

Por otro lado, el análisis de fiabilidad global del cuestionario $s$ EMBU-C ha sido de $\alpha=.673$, lo que coincide con el alfa de Cronbach hallado por otros autores como Canavarro y Pereira (2007), aunque inferior a lo establecido por Márquez-Caraveo, Hernández-Guzmán, Aguilar-Villalobos, Pérez Barrón y Reyes-Sandoval (2007). No obstante, Thondrike (2003) expresa que en ciencias sociales el coeficiente del Alfa de Cronbach por encima de .6 es considerado como aceptable, debido a la naturaleza propia de este tipo de ciencias. 


\subsection{Procedimiento}

Se estableció contacto directo con la dirección de distintos centros educativos a través de una entrevista personal para solicitar su participación en el estudio. No todos accedieron a la aplicación del cuestionario. A aquellos centros educativos que lo permitieron se les facilitó un consentimiento informado que debían firmar las familias antes de su implementación.

\subsection{Análisis de datos}

El análisis de datos se inició con el cálculo de medias y desviaciones típicas para los análisis descriptivos. Posteriormente, se aplicó la prueba Kolmogorov-Smirnov y el test de Levene para comprobar los supuestos de normalidad y homocedasticidad. Los resultados mostraron que era necesario emplear pruebas no paramétricas, por lo que se utilizó el estadístico U de Mann Whitney para variables dicotómicas y el estadístico el H de Kruskal Wallis en variables politómicas. Asimismo cabe destacar la recodificación de una variable que se encontraba codificada de forma inversa en el cuestionario original ("tengo permiso de ir a cualquier lugar que quiera, sin que les importe mucho"). Además se aplicó la $r$ de Pearson para determinar la correlación entre los distintos tipos de estilos educativos de los progenitores.

\section{Resultados}

Los resultados descriptivos evidencian que la dimensión calidez emocional presenta una media considerablemente alta, por lo que los menores perciben una aceptación elevada por parte de sus progenitores. La dimensión rechazo se encuentra por debajo de la media, lo que invita a pensar que los participantes no perciben hostilidad parental. La media de la dimensión sobreprotección se sitúa en la media de la escala, indicando que los padres de los menores encuestados ni son sobreprotectores ni tienen falta de atención hacia sus hijos. La desviación típica de las distintas dimensiones es baja, lo que constata una homogeneidad de respuesta (ver tabla 1). De manera más detallada, las medias y desviaciones típicas de cada uno de los ítems de estas dimensiones quedan expresados en la tabla 2.

Tabla 1

Estadísticos descriptivos de las dimensiones del cuestionario s-EM$B U-C$

\begin{tabular}{lccc}
\hline \multicolumn{1}{c}{ Dimensión } & $\mathrm{N}$ & Media & $\begin{array}{c}\text { Desviación } \\
\text { típica }\end{array}$ \\
\hline Calidez emocional & 163 & 3.2321 & .50662 \\
Rechazo & 164 & 1.2944 & .33706 \\
Sobreprotección & 154 & 2.3052 & .50152 \\
\hline
\end{tabular}

Tabla 2

Estadísticos descriptivos de los ítems del cuestionario s-EMBU-C

\begin{tabular}{|c|c|c|c|}
\hline Ítems & $\mathbf{N}$ & Media & $\begin{array}{l}\text { Desviación } \\
\text { típica }\end{array}$ \\
\hline $\begin{array}{l}\text { 1. Mi(s) padre(s) se enfadan conmi- } \\
\text { go sin decirme la razón }\end{array}$ & 180 & 1.4111 & .69947 \\
\hline 2. $\operatorname{Mi}(\mathrm{s})$ padre(s) me halagan & 178 & 2.7360 & 1.00448 \\
\hline
\end{tabular}

\begin{tabular}{cccc}
\hline Ítems & $\mathrm{N}$ & Media & $\begin{array}{c}\text { Desviación } \\
\text { típica }\end{array}$ \\
\hline
\end{tabular}

3. Deseo que mi(s) padre(s) se preocupen menos de lo que estoy haciendo

$178 \quad 1.5449$

.72929

4. $\mathrm{Mi}(\mathrm{s})$ padre(s) usan castigo físico para castigarme

177

1.3390

.59190

5. Cuando llego a casa, tengo que contarles a mi(s) padre(s) lo que he estado haciendo

$178 \quad 2.7079$

1.02734

6. $\mathrm{Mi}(\mathrm{s})$ padre(s) tratan de hacer mi infancia estimulante, interesante e instructiva (ej. darme libros buenos, organizar que vaya a campamentos y llevarme a actividades deportivas o clubs)

7. Mi(s) padre(s) me critican y me dicen que soy perezoso/a e inútil delante de otros

$180 \quad 1.1389$

8. Mi(s) padre(s) me prohíben hacer cosas que a otros niños o niñas les dejan hacer por miedo de que me pueda pasar algo

9. Mi(s) padre(s) tratan de animarme para llegar a ser el/la mejor

10. Cuando me comporto mal, mi(s) padre(s) tratan de hacerme sentir culpable (por ejemplo, parecen tristes)

11. Mi(s) padre(s) se sienten demasiado ansiosos/as de que algo me pueda pasar

12. $\mathrm{Mi}(\mathrm{s})$ padre(s) tratan de consolarme y animarme si cosas me van mal

13. Me tratan como la oveja negra de la familia

178

1.1517

.45662

14. Mi(s) padre(s) usan palabras y gestos para mostrar que me quieren

$177 \quad 3.2147$

.97071

15. Mi(s) padre(s) quieren más a mis hermanos y/o hermanas que a mí

16. Mi(s) padre(s) me tratan de una manera que me hace sentir avergonzado/a.

$\begin{array}{lll}174 & 1.1724 \quad .53126\end{array}$

17. Tengo permiso de ir a cualquier lugar que quiera, sin que les importe mucho

18. Mi(s) padre(s) se meten en todo lo que hago

19. Hay afecto y ternura entre mi(s) padre(s) y yo

20. Mi(s) padre(s) limitan estrictamente lo que se me permite hacer y no se me permite hacer, y cumplen con estas reglas rigurosamente
$177 \quad 1.1864 \quad .52682$

$\begin{array}{lll}176 & 1.8352 & .82192\end{array}$

$174 \quad 3.2701 \quad .94442$

$\begin{array}{lll}172 & 2.4709 & 1.83031\end{array}$ 


\begin{tabular}{cccc}
\hline \multicolumn{1}{c}{ Ítems } & N & Media & $\begin{array}{c}\text { Desviación } \\
\text { típica }\end{array}$ \\
\hline $\begin{array}{l}\text { 21. Mi(s) padre(s) me castigan du- } \\
\text { ramente, aun en el caso de ha- } \\
\text { ber hecho una tontería }\end{array}$ & 175 & 1.4457 & .73981 \\
$\begin{array}{l}\text { 22. Mi(s) padre(s) quieren decidir } \\
\text { cómo debo vestirme o cómo } \\
\text { debo parecer }\end{array}$ & 176 & 2.1477 & 1.04788 \\
$\begin{array}{l}\text { 23. Mi(s) padre(s) están orgullo- } \\
\text { sos/as cuando tengo éxito en } \\
\text { algo que he emprendido }\end{array}$ & 178 & 3.6180 & .67239 \\
\hline
\end{tabular}

Posteriormente, se aplicó una correlación de Pearson para determinar la posible relación entre las tres variables del cuestionario. Se evidenció la existencia de correlación negativa y muy baja entre las variables rechazo y calidez emocional (-.187), y positiva y baja entre las variables rechazo y sobreprotección (.393), por lo que no se pueden determinar resultados concluyentes al respecto.

Por otro lado, los resultados del cuestionario EU Kids online II mostraron que los menores afirman que sus padres conocen lo que hacen en Internet (conocimiento parental). Un $35.4 \%$ creen que mucho, un $29.1 \%$ consideran que bastante, un $17.7 \%$ opinan que poco y un $4.4 \%$ afirman que nada. No obstante, un 13.1 no son capaces de percibir lo que conocen sus progenitores.

En la pregunta "¿te gustaría que tu(s) padre(s) se interesasen más o menos en lo que haces en Internet, o que siguiese igual?" (interés parental), el 52.9\% de los menores preferirían que este interés siguiese igual, el 10.5\% desearía que sus padres se interesasen mucho más y el $9.3 \%$ solo un poco más. Sin embargo, el $8.7 \%$ opina que le gustaría que se interesasen un poco menos y el mismo porcentaje resultaría para quienes preferirían que este interés fuera mucho menos. Nuevamente resulta llamativo que un $9.9 \%$ afirma no saber que preferiría.

Ante la pregunta ¿las cosas que hacen tus padres en relación a cómo usas Internet te ayudan a hacer que tu experiencia en Internet sea mejor?, los resultados muestran que la acción parental es muy valorada por los menores. Un $42.6 \%$ considera que las acciones de los progenitores les ayudan mucho, un $26.1 \%$ solo un poco, un $10.8 \%$ no les ayuda nada y un $20.5 \%$ no sabe responder a la pregunta.

Al preguntar a los menores si tienen en cuenta lo que sus padres les han dicho al usar Internet (opinión parental), un 72.2\% reconoce que sí lo hacen mucho, un $14.8 \%$ solo un poco, un $5.1 \%$ no lo tienen presente y un $8 \%$ no sabe qué responder.

Cuando los menores responden a la pregunta "¿hacen ahora tus padres algo nuevo o diferente por haberte molestado o disgustado algo de Internet?, resulta alarmante que solo un $14.9 \%$ reconoce nuevas acciones parentales, el $43.4 \%$ afirma que sus padres no hacen nada nuevo y el $41.7 \%$ no sabe qué contestar.

Junto a estas preguntas, el cuestionario EU Kids online II fija cinco tipos de mediación familiar (mediación activa del uso de Internet o co-uso, mediación restrictiva, mediación restrictiva de seguridad en Internet, monitorización y, finalmente, mediación técnica). Los estadísticos descriptivos aparecen recogidos en las tablas 3 y 4.

La mediación basada en el co-uso, es decir, todo tipo de actividades de medios compartidos por padres e hijos, en 4 de los 5 ítems, los menores afirman mayoritariamente que este tipo de mediación no se produce en sus hogares. De igual forma, la monitorización (controlar o revisar las actividades en línea de los menores después de su actividad) no es una estrategia que suelan emplear las familias en su relación con los medios, al igual que la mediación técnica, entendida como restringir el contenido en línea o el tiempo de permanencia en línea aplicando prohibiciones o filtros técnicos. En este último tipo de mediación los menores expresan que sus padres sí usan programas para prevenir correos no deseados o virus.

Por el contrario, la mediación activa de seguridad en Internet es la estrategia más utilizada por los progenitores, lo que implica que los estos explican y discuten el contenido de los medios a los que acceden sus hijos.

La mediación restrictiva, es decir, establecer reglas sobre dónde, cuándo, por cuánto tiempo y a qué se debe acceder, se produce en determinados aspectos muy concretos como es tener su propio perfil en una red social y subir fotos, vídeos o música para compartir con otros.

Las familias muestran una mayor permisividad en el uso de sistemas de mensajería, en la descarga de música o películas, así como la visualización de vídeos en Youtube.

Tabla 3

Porcentajes de respuesta en los tipos de mediación co-uso, mediación activa de seguridad en internet, monitorización y mediación técnica

\begin{tabular}{|c|c|c|c|c|}
\hline Dimensión & Ítems & Sí & No & No sé \\
\hline \multirow{5}{*}{$\begin{array}{l}\text { Dirías que tu } \\
\text { padre / tus } \\
\text { padres, a ve- } \\
\text { ces... }\end{array}$} & $\begin{array}{l}\text { Habla contigo sobre o } \\
\text { que haces en Internet }\end{array}$ & 44.3 & 47.7 & 8 \\
\hline & $\begin{array}{l}\text { Se sienta contigo mien- } \\
\text { tras usas Internet (mi- } \\
\text { rando lo que haces, pero } \\
\text { sin participar realmente) }\end{array}$ & 27.7 & 59 & 13.3 \\
\hline & $\begin{array}{l}\text { Está cerca cuando usas } \\
\text { Internet }\end{array}$ & 56.1 & 32.4 & 11.6 \\
\hline & $\begin{array}{l}\text { Te anima a explorar y } \\
\text { aprender cosas por tu } \\
\text { cuenta en Internet }\end{array}$ & 35.4 & 48 & 16.6 \\
\hline & $\begin{array}{l}\text { Comparte actividades } \\
\text { contigo en Internet }\end{array}$ & 31.6 & 58 & 10.3 \\
\hline \multirow[b]{3}{*}{$\begin{array}{l}\text { Mediación } \\
\text { activa de se- } \\
\text { guridad en } \\
\text { Internet }\end{array}$} & $\begin{array}{l}\text { Ayudarte cuando tienes } \\
\text { dificultad para encontrar } \\
\text { algo en Internet }\end{array}$ & 81.3 & 13.1 & 5.7 \\
\hline & $\begin{array}{l}\text { Explicar por qué algunas } \\
\text { páginas web son buenas } \\
\text { o son malas }\end{array}$ & 77.3 & 14.2 & 8.5 \\
\hline & $\begin{array}{l}\text { Aconsejarte sobre cómo } \\
\text { usar Internet de forma } \\
\text { segura }\end{array}$ & 80.6 & 13.1 & 6.3 \\
\hline \multirow{3}{*}{$\begin{array}{l}\text { ¿Ha hecho } \\
\text { algunos de tus } \\
\text { padres alguna } \\
\text { vez alguna de } \\
\text { las siguientes } \\
\text { cosas? }\end{array}$} & $\begin{array}{l}\text { Sugerir maneras de com- } \\
\text { portamiento hacia otras } \\
\text { personas en la red }\end{array}$ & 39.2 & 35.8 & 25 \\
\hline & $\begin{array}{l}\text { Ayudarte, cuando algo, } \\
\text { en Internet, te ha preo- } \\
\text { cupado o disgustado }\end{array}$ & 56 & 23.4 & 20.6 \\
\hline & $\begin{array}{l}\text { En general, hablar con- } \\
\text { tigo sobre lo que tienes } \\
\text { que hacer si algo, en } \\
\text { Internet, te disgustase o } \\
\text { molestase }\end{array}$ & 59.4 & 20.6 & 20 \\
\hline
\end{tabular}




\begin{tabular}{|c|c|c|c|c|}
\hline Dimensión & Ítems & Sí & No & No sé \\
\hline \multirow{4}{*}{$\begin{array}{l}\text { Monitorización } \\
\text { Cuando usas } \\
\text { Internet en } \\
\text { casa, ¿tu pa- } \\
\text { dre/ alguno } \\
\text { de tus padres } \\
\text { comprueban } \\
\text { alguna vez } \\
\text { alguna de las } \\
\text { siguientes } \\
\text { cosas? }\end{array}$} & $\begin{array}{l}\text { Las páginas que has vi- } \\
\text { sitado }\end{array}$ & 32.4 & 41 & 26.6 \\
\hline & $\begin{array}{l}\text { Los mensajes en tu co- } \\
\text { rreo o cuenta de men- } \\
\text { sajería instantánea } \\
\text { (Messenger, Whatsapp, } \\
\text { Hangouts) }\end{array}$ & 30.1 & 53.2 & 16.8 \\
\hline & $\begin{array}{l}\text { Tu perfil en una red } \\
\text { social (por ejemplo, } \\
\text { Instagram, Facebook, } \\
\text { Snapchat, Twitter, etc.) o } \\
\text { comunidad online }\end{array}$ & 25.3 & 51.2 & 23.5 \\
\hline & $\begin{array}{l}\text { Qué amigos o contac- } \\
\text { tos añades a tu perfil de } \\
\text { red social (por ejemplo, } \\
\text { Instagram, Facebook, } \\
\text { Snapchat, Twitter, etc.) } \\
\text { o servicio de mensajería } \\
\text { instantánea (Messenger, } \\
\text { Whatsapp, Hangouts) }\end{array}$ & 33.5 & 41 & 25.4 \\
\hline \multirow{4}{*}{$\begin{array}{c}\text { Mediación } \\
\text { técnica } \\
\text { ¿Hacen } \\
\text { tus padres } \\
\text { algunas de } \\
\text { las siguientes } \\
\text { cosas en el } \\
\text { dispositivo que } \\
\text { tú usas más } \\
\text { a menudo en } \\
\text { casa? }\end{array}$} & $\begin{array}{l}\text { Controles parentales } u \\
\text { otro tipo de programas, } \\
\text { para bloquear algunos } \\
\text { tipos de páginas web } \\
\text { (programas que NO per- } \\
\text { miten que visites ciertas } \\
\text { páginas o evita cierto } \\
\text { tipo de actividades en } \\
\text { Internet) }\end{array}$ & 23.7 & 45.1 & 30.6 \\
\hline & $\begin{array}{l}\text { Controles parentales u } \\
\text { otro tipo de software } \\
\text { para hacer seguimiento } \\
\text { de las páginas web vi- } \\
\text { sitadas (programas que } \\
\text { graban las páginas web } \\
\text { que has visitado para } \\
\text { poder comprobar des- } \\
\text { pués qué has hecho en } \\
\text { Internet) }\end{array}$ & 16.7 & 55.7 & 21.6 \\
\hline & $\begin{array}{l}\text { Tener un servicio o con- } \\
\text { trato que limita el tiem- } \\
\text { po que pasas en Internet }\end{array}$ & 17.3 & 60.1 & 22.5 \\
\hline & $\begin{array}{l}\text { Programas para preve- } \\
\text { nir correos no deseados } \\
\text { o virus }\end{array}$ & 43.4 & 32.4 & 24.3 \\
\hline
\end{tabular}

Con el fin de comprobar las relaciones existentes entre las distintas variables del cuestionario s EMBU-C y las variables sociodemográficas, así como las variables vinculadas a las actuaciones parentales complementarias a la mediación y los distintos ítems de los tipos de mediación, se optó por realizar un análisis de contraste de hipótesis mediante el uso de pruebas no paramétricas (ver tabla 5).

En la dimensión Calidez emocional, la variable género establece diferencias estadísticamente significativas entre chicos y chicas, aquellos perciben una menor calidez emocional de sus progenitores que las chicas. Por su parte, la variable acciones parentales también muestra diferencias significativas. Para comparar entre qué grupos se establecían dichas diferencias se aplicó la prueba de U Mann-Withney, asimismo, se consideró realizar un ajuste en el nivel de significatividad (corrección de Bonferroni) para contrarrestar el incremento en la tasa de error tipo I. Por tanto, la significatividad quedó establecida en $\mathrm{p}=.013(.05 / 4)$ para los grupos que consideraban que las cosas que hacían progenitores en relación a cómo usaban Internet les ayudaban mucho a mejorar la experiencia con Internet y los que afirmaban que estas cosas no les ayudaba. Los primeros perciben una mayor calidez emocional de sus progenitores que los segundos (ver tabla 6).

Las variables que ofrecen un valor significativo en la dimensión Sobreprotección, fueron "la revisión por parte de los progenitores de las páginas web que visitan los menores" (Mediación-monitorización), las acciones parentales y la limitación parental. La aplicación de la prueba de U Mann-Withney y la corrección de Bonferroni fijó una significatividad de $\mathrm{p}=.017(.05 / 3)$ en el primer caso y de $\mathrm{p}=.013(.05 / 4)$ en los dos restantes.

Los menores que afirman que sus progenitores revisan las páginas web que consultan perciben una mayor sobreprotección de sus padres que los que niegan esta consulta (Mediación-monitorización). Por otro lado, los menores que consideraban que las cosas que hacían progenitores en relación a cómo usaban Internet les ayudaban un poco a mejorar la experiencia con Internet, frente a los que no sabían responder a la respuesta, muestran un mayor grado de percepción de sobreprotección. Por su parte, los menores que responden que las actuaciones parentales limitan mucho sus acciones en Internet sienten un mayor grado de sobreprotección de sus padres que los que niegan esta limitación o no saben responder a la pregunta (ver tabla 7).

Tabla 4

Porcentajes de respuesta en la mediación restrictiva

\begin{tabular}{|c|c|c|c|c|c|}
\hline Dimensión & Ítems & Me lo permiten & $\begin{array}{l}\text { Me lo permiten con } \\
\text { permiso o supervisión }\end{array}$ & $\begin{array}{l}\text { Nunca me lo } \\
\text { permiten }\end{array}$ & No sé \\
\hline & Usar el Messenger, Whatsapp o Hangouts & 48.6 & 22.5 & 19.7 & 9.2 \\
\hline \multirow{2}{*}{$\begin{array}{l}\text { Mediación } \\
\text { restrictiva }\end{array}$} & Bajarme música o películas de Internet & 43.7 & 27 & 13.8 & 15.5 \\
\hline & Ver vídeoclips en Internet (por ejemplo, en YouTube) & 69.9 & 19.3 & 6.8 & 4 \\
\hline \multirow{3}{*}{$\begin{array}{c}\text { Tus padres } \\
\text { en la ac- } \\
\text { tualidad te } \\
\text { permiten } \\
\ldots\end{array}$} & $\begin{array}{l}\text { Tener tu propio perfil en una red social (por ejemplo, } \\
\text { Instagram, Facebook, Snapchat, Twitter, etc.) }\end{array}$ & 18.3 & 28 & 42.3 & 11.4 \\
\hline & $\begin{array}{l}\text { Dar información personal a otros por Internet (por } \\
\text { ejemplo el nombre completo, dirección o número de } \\
\text { teléfono) }\end{array}$ & 7.4 & 10.3 & 72.6 & 9.7 \\
\hline & $\begin{array}{l}\text { Subir fotos, vídeos o música para compartir con } \\
\text { otros }\end{array}$ & 21.8 & 27.6 & 37.6 & 12.9 \\
\hline
\end{tabular}


Tabla 5

Contraste de hipótesis entre variables.

\begin{tabular}{|c|c|c|c|c|c|}
\hline Dimensión & Variables & $\begin{array}{l}\text { U de Mann- } \\
\text { Whitney }\end{array}$ & $\begin{array}{l}\text { Kruskal-Wallis } \\
\text { Chi-cuadrado (gl) }\end{array}$ & Sig. & Rango promedio \\
\hline \multirow{8}{*}{ Calidez emocional } & \multirow{8}{*}{ Acciones parentales } & \multirow{8}{*}{2575.500} & & \multirow{3}{*}{.015} & Chicos=73.77 \\
\hline & & & & & \\
\hline & & & & & Chicas=91.66 \\
\hline & & & & & Sí mucho=91.29 \\
\hline & & & & & Sí, un poco $=80.25$ \\
\hline & & & $11.940(3)$ & .008 & \\
\hline & & & & & \\
\hline & & & & & No sé=74.86 \\
\hline \multirow{12}{*}{ Sobreprotección } & \multirow{2}{*}{$\begin{array}{l}\text { Mediación -Monitori- } \\
\text { zación }\end{array}$} & & \multirow{3}{*}{$9.412(2)$} & \multirow{3}{*}{.009} & Sí $=86.30$ \\
\hline & & & & & $\mathrm{No}=63.16$ \\
\hline & \multirow[t]{2}{*}{ Páginas que visita } & & & & No sé $=83.25$ \\
\hline & & & \multirow{5}{*}{$9.966(3)$} & \multirow{5}{*}{.019} & Sí mucho=80.63 \\
\hline & \multirow{4}{*}{ Acciones parentales } & & & & Sí, un poco $=86.68$ \\
\hline & & & & & \\
\hline & & & & & $\mathrm{No}=56.53$ \\
\hline & & & & & No sé $=60.06$ \\
\hline & \multirow{4}{*}{ Limitación parental } & & \multirow{4}{*}{$7.884(3)$} & \multirow{4}{*}{.048} & Sí, mucho=93.61 \\
\hline & & & & & Sí, un poco $=77.35$ \\
\hline & & & & & \\
\hline & & & & & No sé $=68.07$ \\
\hline \multirow{8}{*}{ Rechazo } & \multirow{5}{*}{ Edad } & & \multirow{5}{*}{$12.821(3)$} & \multirow{4}{*}{.005} & 10 años $=53.53$ \\
\hline & & & & & 11 años $=86.36$ \\
\hline & & & & & 12 años $=88.09$ \\
\hline & & & & & 13 años $=24.50$ \\
\hline & & & & \multirow{4}{*}{.012} & Sí, mucho=73.75 \\
\hline & \multirow{3}{*}{ Opinión parental } & & \multirow{3}{*}{ 11.0.38 (3) } & & Sí, un poco=97.12 \\
\hline & & & & & $\mathrm{No}=114$ \\
\hline & & & & & No sé $=85.91$ \\
\hline
\end{tabular}

Tabla 6

Rangos y estadísticos de prueba Dimensión Calidez emocional

\begin{tabular}{|c|c|c|c|c|c|c|c|}
\hline \multirow[b]{2}{*}{ Variables } & \multicolumn{3}{|c|}{ Rangos } & \multicolumn{4}{|c|}{ Estadísticos de prueba } \\
\hline & $\mathbf{N}$ & Rango promedio & Suma de rangos & $\begin{array}{c}\text { U de } \\
\text { Mann-Whitney }\end{array}$ & W de Wilcoxon & $\mathbf{Z}$ & $\mathrm{p}$ \\
\hline \multicolumn{8}{|l|}{ Género } \\
\hline Chica & 75 & 91.66 & 6874.50 & 2575.500 & 6491.500 & -2.428 & .015 \\
\hline Chico & 88 & 73.77 & 6.491 .50 & & & & \\
\hline Acciones parentales & & & & 308.000 & 479.000 & -3.250 & .001 \\
\hline Sí, mucho & 68 & 47.97 & 3262.000 & & & & \\
\hline No & 18 & 26.61 & 479.00 & & & & \\
\hline
\end{tabular}


Tabla 7

Rangos y estadísticos de prueba Dimensión Sobreprotección

\begin{tabular}{|c|c|c|c|c|c|c|c|}
\hline \multirow[t]{2}{*}{ Variables } & \multicolumn{3}{|c|}{ Rangos } & \multicolumn{4}{|c|}{ Estadísticos de prueba } \\
\hline & $\mathbf{N}$ & Rango prome-dio & Suma de rangos & $\begin{array}{c}\text { U de } \\
\text { Mann-Whitney }\end{array}$ & W de Wilcoxon & $\mathrm{Z}$ & $\mathrm{p}$ \\
\hline Mediación-monitori-zación & & & & 1058.000 & 3203.000 & -2.776 & .006 \\
\hline Sí & 47 & 66.49 & 3125.00 & & & & \\
\hline No & 65 & 49.28 & 3203.00 & & & & \\
\hline Acciones parentales & & & & 345.500 & 723.500 & -2.497 & .013 \\
\hline Sí, un poco & 33 & 38.86 & 1554.50 & & & & \\
\hline No sé & 43 & 26.80 & 723.50 & & & & \\
\hline \multicolumn{8}{|l|}{ Limitación parental } \\
\hline Sí, mucho & 33 & 46.14 & 1522.50 & \multirow{2}{*}{457.500} & \multirow{2}{*}{1403.500} & \multirow{2}{*}{-2.649} & \multirow{2}{*}{.008} \\
\hline No & 43 & 32.64 & 1403.50 & & & & \\
\hline Sí, mucho & 33 & 44.52 & 1469.00 & \multirow{2}{*}{445.000} & \multirow{2}{*}{1306.000} & \multirow{2}{*}{-2.526} & \multirow{2}{*}{.012} \\
\hline No sé & 41 & 31.85 & 1306.00 & & & & \\
\hline
\end{tabular}

En la dimensión Rechazo se aprecia que a mayor edad de los niños, estos perciben en mayor grado el rechazo por parte de sus progenitores (ver tabla 8). Por otro lado, esta percepción de rechazo es también mayor en aquellos niños que tienen muy en cuenta lo que sus padres le dicen al usar Internet, que en aquellos otros que no consideran la opinión de sus padres.

\section{Discusión de los resultados y conclusiones}

La tradicional clasificación de estilos parentales (Baumrind, 1966) y adaptaciones posteriores requieren de una revisión que se ajuste a una sociedad global, líquida y digitalizada en la que muchos de los comportamientos parentales se diluyen, también influidos por la generalización del uso de dispositivos móviles y la conectividad que posibilitan.

En el contexto de investigación, la calidez emocional y la protección moderada caracterizan las prácticas educativas de los progenitores de los participantes. Esta calidez emocional es percibida mayormente por las chicas y por aquellos menores cuyos progenitores hacen cosas que les ayuda mucho a mejorar la experiencia en Internet.
Los menores que perciben una mayor limitación de sus acciones en Internet por parte de sus padres, observan un mayor nivel de sobreprotección. Los resultados evidencian también que, a mayor edad de los menores, estos perciben un mayor rechazo de sus progenitores. Las características evolutivas de los menores en estas edades pueden ser una explicación lógica, ya que se sitúan en la preadolescencia. Sin embargo, resulta paradójico que aquellos menores que tienen en cuenta la opinión de sus padres al usar Internet son también los que perciben más rechazo de estos. Sería necesario indagar estos aspectos psicológicos y determinar por qué se producen estas incongruencias. Asimismo, la investigación no ha podido demostrar totalmente la asociación entre las prácticas educativas y las distintas modalidades de mediación parental.

El análisis de la mediación percibida por los menores sitúa su respuesta en la línea de diferentes investigaciones y la ubica en aquellas que han distinguido un mayor uso de estrategias activas (Garmendia, Garitaonandia, Martínez y Casado, 2012). Sin embargo, también se aprecia un uso mixto de estrategias en función de la acción que se desarrolle. Así, los menores cuyos padres ayudan a tener una mejor experiencia en Internet recono-

Tabla 8

Rangos y estadísticos de prueba Dimensión Rechazo

\begin{tabular}{|c|c|c|c|c|c|c|c|}
\hline \multirow[b]{2}{*}{ Variables } & \multicolumn{3}{|c|}{ Rangos } & \multicolumn{4}{|c|}{ Estadísticos de prueba } \\
\hline & $\mathbf{N}$ & $\begin{array}{c}\text { Rango } \\
\text { promedio }\end{array}$ & $\begin{array}{c}\text { Suma } \\
\text { de rangos }\end{array}$ & U de Mann-Whitney & $\begin{array}{c}\text { W de } \\
\text { Wilcoxon }\end{array}$ & $\mathbf{Z}$ & $\mathbf{p}$ \\
\hline \multicolumn{8}{|l|}{ Edad } \\
\hline 10 años & 17 & 32.09 & 545.50 & \multirow[t]{2}{*}{392.500} & \multirow[t]{2}{*}{545.500} & \multirow[t]{2}{*}{-2.809} & \multirow[t]{2}{*}{.005} \\
\hline 11 años & 80 & 52.59 & 4207.50 & & & & \\
\hline 10 años & 17 & 28.24 & 480.00 & \multirow[t]{2}{*}{327.000} & \multirow[t]{2}{*}{480.000} & \multirow[t]{2}{*}{$-2,590$} & \multirow[t]{2}{*}{.010} \\
\hline 12 años & 64 & 44.39 & 2841.00 & & & & \\
\hline Opinión parental & & & & 308.000 & 479.000 & -3.250 & .001 \\
\hline Sí, mucho & 115 & 60.34 & 6939.00 & \multirow{2}{*}{269.000} & \multirow{2}{*}{6939.000} & \multirow{2}{*}{-2.468} & \multirow{2}{*}{.014} \\
\hline No & 9 & 90.11 & 811.00 & & & & \\
\hline
\end{tabular}


cen una mayor sobreprotección de sus progenitores en acciones como revisar las páginas web que consultan (monitorización). La aplicación mixta de estrategias obedecería posiblemente a las diferentes definiciones del concepto de mediación y sus distintas clasificaciones (Livingstone y Helsper, 2008). Recientemente, el mismo cuestionario EU Kids Online ha sido redefinido e incorpora una nueva clasificación (habilitante, filial, restrictiva y técnica) (Garmendia et al. 2019).

De forma complementaria a las prácticas educativas y a las estrategias de mediación, los progenitores, a juicio de sus hijos, realizan determinadas acciones que permiten la gestión de los medios e Internet en el hogar. Los menores manifiestan que sus padres conocen lo que hacen en Internet, les gusta que estén interesados en ello, que les ayuden a mejorar la relación con la Red y tienen muy en cuenta lo que sus progenitores les comentan sobre esta.

No obstante, el 43\% de los menores encuestados afirman que después de haber comunicado a los progenitores que algo les molesta o les disgusta en Internet, estos no hacen nada, esto podría deberse a falta de conocimiento o estrategia. Aspecto que contrasta con la preocupación que suelen manifestar los padres en diferentes investigaciones (Garmendia et al., 2019). Para conocer los motivos hubiera convenido aplicar el cuestionario a las familias también e indagar sobre la competencia mediática de los progenitores (González-Fernández, Ramírez-García y Aguaded, 2019).

Por último, sería deseable avanzar en identificar aquellas estrategias de mediación parental más efectivas para un uso positivo de los medios, que determinar el tipo de mediación que tiene lugar en los hogares.

\section{REFERENCIAS BIBLIOGRÁFICAS}

Aierbe, A. Orozco, G., y Medrano, C. (2014). Family context, television and perceived values. A cross-cultural study with adolescents. Communication and Society, 27(2), 79-99. Recuperado de https://goo.gl/am61yV

Arrindell, W. A., Sanavio, E., Aguilar, G., Sica, C., Hatzichristou, C., Eisemann, M., y Kállai, J. (1999). The development of a short form of the EMBU1: Its appraisal with students in Greece, Guatemala, Hungary and Italy. Personality and individual Differences, 27(4), 613-628.

Bartau-Rojas, I., Aierbe-Barandiaran, A., y Oregui-González, E. (2018). Mediación parental del uso de Internet en el alumnado de Primaria: creencias, estrategias y dificultades. Comunicar, 54, 71-79. https: / / doi.org/10.3916/C54-2018-07.

Baumrind, D. (1966). Effects of Authoritative Parental Control on Child Behavior. Child Development, 37(4), 887-907.

Baumrind, D. (1991). The influence of parenting style on adolescent competence and substance abuse. Journal of Early Adolescence, 11, 56-95.

Brito, R., Francisco, R., Dias, P., y Chaudron, S. (2017). Family dynamics in digital homes: The role played by parental mediation in young children's digital practices around 14 European countries. Contemporary Family Therapy, 39, 271-280. https:/ / doi.org/10.1007/s10591-017-9431-0

Cabrera, V.E., y Salazar, P.A. (2014). Estilos de vida de los jóvenes y las necesidades de educación sexual. Bogotá: Instituto de Estudios del Ministerio Público.

Canavarro, M.C., y Pereira, A.I. (2007). A percepção dos filhos sobre os estilos educativos parentais: A versão portuguesa do EMBU-C. Revista Iberoamericana de Diagnóstico y Evaluación / E Avaliação Psicológica, 24, 192-210.

Capano, A., Tornaría, G., del Luján, M., y Massonnier, N. (2016). Estilos relacionales parentales: estudio con adolescentes y sus padres. Revista de Psicología (PUCP), 34(2), 413-444. http:/ / dx.doi.org/10.18800/psico.201602.008

Ceballos, E., y Rodrigo, M. J. (1998). Las metas y estrategias de socialización entre padres e hijos. En M. Rodrigo, y J. Palacios, Familia y desarrollo humano (pp. 225-243). Madrid: Alianza.

Chorot, P., Valiente, R. M., Magaz, A. M., Santed, M. A., y Sandin, B. (2017). Perceived parental child rearing and attachment as predictors of anxiety and depressive disorder symptoms in children: The mediational role of attachment. Psychiatry research, 253, 287-295. http://dx.doi.org/10.1016/j.psychres.2017.04.015

Claesson, K., y Sohlberg, S. (2002). Internalized shame and early interactions characterized by indifference, abandonment and rejection: Replicated findings. Clinical Psychology \& Psychotherapy, 9(4), 277-284.

Cuero, J. C., Gutiérrez, P., e Infante, A. (2015). La familia, un vínculo duradero cimentado en el afecto: creencias sobre familia de los jóvenes escolarizados de colegios en convenio de Bogotá. Actualidades Pedagógicas, 65, 49-67. http://dx.doi. org/10.19052/ap.3059

Daneels, R., y Vanwynsberghe, H. (2017). Mediating social media use: Connecting parents' mediation strategies and social media literacy. Cyberpsychology: Journal of Psychosocial Research on Cyberspace, 11(3), article 5. http://dx.doi.org/10.5817/ CP2017-3-5

Deslandes, R. (2019). A framework for school-family collaboration integrating some relevant factors and processes. Aula Abierta, 48, 2, 11-19

Duggan, M., Lenhart, A., Lampe, C., y Ellison, N.B. (2015). Parents and Social Media. Washington, DC: Pew Research Centre Report: Internet \& Technology. Recuperado de https:/ /goo. $\mathrm{gl} / \mathrm{AMXm7G}$

Dürager, A. y Sonck, N. (2014). Testing the reliability of scales on parental internet mediation. EU Kids Online, LSE, London, UK. Recuperado de https:/ / bit.ly/2EWmJrw

Fletcher, A.C., y Blair, B.L. (2014). Maternal authority regarding early adolescents' social technology use. Journal of Family Issues, 35(1), 54-74.https: / / doi.org/10.1177/0192513X12467753

Fundación Telefónica (2019). Sociedad Digital en España. Recuperado de https: / bit.ly/2MvKGtU

García, M., Pelegrina, S., y Lendínez, J. (2002). Los estilos educativos de los padres y la competencia psicosocial de los adolescentes. Anuario de Psicología, 33, 79-95.

Garmendia, M., Casado, M.A., Martínez, G., y Garitaonandia, C. (2013). Las madres y padres, los menores e Internet: Estrategias de mediación parental en España. Doxa, 17, 99-117. Recuperado de https:/ / goo.gl/HbZyKZ

Garmendia, M., Jiménez, E., Karrera, I., Larrañaga, N., Casado, M.Á., Martínez, G., y Garitaonandia, C. (2019). Actividades, mediación, oportunidades y riesgos online de los menores en la era de la convergencia mediática. León: INCIBE.

Gentile, D. A., Nathanson, A. I., Rasmussen, E. E., Reimer, R. A., y Walsh, D. A. (2012). Do you see what I see? Parent and child reports of parental monitoring of media. Family Relations, 61, 470-487. https://doi.org/10.1111/j.1741-3729.2012.00709.x

Giménez, A.M., Luengo, J.A., y Bartrina, M.J. (2017). ¿Qué hacen los menores en Internet? Usos de las TIC, estrategias de supervisión parental y exposición a riesgos. Electronic Journal of Research in Educational Psychology, 15(3), 533-552. https:/ / dx.doi.org/10.14204/ejrep.43.16123

González-Fernández, N., Ramírez-García, A., y Aguaded, I. (2019). Alfabetización mediática en escenarios familiares. Diagnóstico, necesidades y propuesta formativa. Education in 
the Knowledge Society, 20, 1- 13. http:/ /dx.doi.org/10.14201/ eks2019_20_a11

Han, S. Y., y Kim, Y. H. (2012). Interpersonal rejection experiences and shame as predictors of susceptibility to peer pressure among Korean children. Social Behavior and Personality: an international journal, 40(7), 1213-1231.

Helsper, E. J., Kalmus, V., Hasebrink, U., Sagvari, B., y de Haan, J. (2013). Country classification: opportunities, risks, harm and parental mediation. London: EU Kids Online, The London School of Economics and Political Science.

Instituto Nacional de Estadística (2019). Equipamiento y Uso de Tecnologías de la Información y Comunicación en los hogares españoles. Recuperado de https:/ / bit.ly/2PYxno3

Jorge, E., y González, M. C. (2017). Estilos de crianza parental: una revisión teórica. Informes Psicológicos, 17(2), 39-66.

Key Takeaways from Mary Meeker's 2019 Internet Trends Report. Recuperado de https:/ / bit.ly/2Q1BpfE

Lauricella, A.R., Wartella, E.A., y Rideout, V.J. (2015). Young children's screen time: The complex role of parent and child factors. Journal of Applied Developmental Psychology, 36, 11-17. https:/ / doi.org/10.1016/j.appdev.2014.12.001

Liu, Y., Fei, L., Sun, X., Wei, C., Luo, F., Li, Z., y Lin, X. (2018). Parental Rearing Behaviors and Adolescent's Social Trust: Roles of Adolescent Self-esteem and Class Justice Climate. Journal of Child and Family Studies, 27(5), 1415-1427.

Livingstone, S., y Haddon, L. (2009). EU Kids Online: Final report. LSE, London: EU Kids Online. Recuperado de https://bit. ly/2ZqOUZa

Livingstone, S., y Helsper, E. (2008). Parental mediation and children's Internet use. Journal of Broadcasting E Electronic Media, 52(4), 581-599. https: / / doi.org/10.1080/08838150802437396

Livingstone, S., Mascheroni, G., Dreier, M., Chaudron, S., y Lagae, K. (2015). How parents of young children manage digital devices at home: The role of income, education and parental style. Recuperado de https: / / bit.ly/2MzS9bt

Marciales, G.P., y Cabra, F. (2011). Internet y pánico moral: revisión de la investigación sobre la interacción de niños y jóvenes con los nuevos medios. Universitas Psychologica, 10(3), 855-865. Recuperado de https://goo.gl/Ufs9tf

Márquez-Caraveo, M.E., Hernández-Guzmán, L., Aguilar-Villalobos, J., Pérez Barrón, V., y Reyes-Sandoval, M. (2007). Datos psicométricos del EMBU-I "Mis memorias de crianza" como indicador de la percepción de crianza en una muestra de adolescentes de la Ciudad de México. Salud Mental, 20, 58-66.

Martínez de Morentin, J.I., y Medrano, C. (2012). La mediación parental y el uso de internet. International Journal of Developmental and Educational Psychology, 1(1), 549-556. Recuperado de https://goo.gl/vop4Gk

Martínez-Vasallo, H. M. (2015). La familia: una visión interdisciplinaria. Revista médica electrónica, 37(5), 523-534.

Monks, C.P., Mahdavi, J., y Rix, K. (2016). The emergence of cyberbullying in childhood: Parent and teacher perspectives. Psicología Educativa, 22, 39-48. https://doi.org/10.1016/j. pse.2016.02.002
Nikken, P., y Jansz, J. (2006). Parental mediation of children's videogame playing: A comparison of the reports by parents and children. Learning, Media and Technology, 31, 181-202. https:/ / doi.org/10.1080/17439880600756803

ONTSI (2019). Las TIC en los hogares españoles. Tercer trimestres 2018. Recuperado de https:/ / bit.ly/34X5CjX

Penelo, E., Viladrich, M. C., y Doménech, J. M. (2009). Cuestionarios de estilo educativo percibido por niños (EMBU-C), adolescentes (EMBU-A) y progenitores (EMBU-P). Barcelona: Universitat Autònoma de Barcelona.

Ramírez-García, A., Salcines-Talledo, I., y González-Fernández, N. (2020). Los dispositivos móviles en el hogar. Interés formativo de las familias españolas. Revista Española de Orientación y Psicopedagogía, 31(1), 43-61. https://doi.org/10.5944/ reop.vol.31.num.1.2020.27286

Rodríguez-Ruiz, B., Álvarez-Blanco, L., Martínez-González, R.A. y Epstein J. (2019). Relación entre Centros Educativos, Familias y Entidades Comunitarias, Aula Abierta, 48, 2, 7-11

Thondrike, R.L (2003). Medición y evaluación psicológica en Educación. México: Trillas.

Valcke, M., De-Weber, B., Van-Keer, H., y Schellens, T. (2011). Long-term study on safe Internet use of young children. Computers \& Education, 57(1), 1292-1305. https://doi.org/10.1016/j.compedu.2011.01.010

Symons, K., Ponnet, K., Emery, K., Walrave, M., y Heirman, W. (2017). A factorial validation of parental mediation strategies with regard to internet use. Psychologica Belgica, 57, 93-111. https: / / doi.org/10.5334/pb.372

Sonck, N., Nikken, P., de Haan, J. (2013) Determinants of internet mediation. Journal of Children and Media, 7(1), 96-113.

Torrecillas-Lacave, T., Vázquez-Barrio, T., Suárez, R., y Fernández-Martínez, L.M. (2020). El papel de los padres en el comportamiento online de menores hiperconectados. Revista Latina de Comunicación Social, 75, 121-148. https://doi. org/10.4185/RLCS-2020-1419

Valcke M., Bonte S., De Wever B., y Rots I. (2010). Internet parenting styles and the impact on Internet use of primary school children. Computer \& Education, 55, 454-464. DOI: 10.1016/j. compedu.2010.02.009.

Wartella, E., Rideout, V., Lauricella, A., y Connell, S. (2013). Parenting in the age of digital technology. Report for the Center on Media and Human Development. Illinois, USA: Northwestern University. Recuperado de https://goo.gl/MHVFQn

Wilfred, W., y Allan, H. (2016). The relative importance of paternal and maternal parenting as predictors of adolescents' home Internet use and usage. Computers E Education, 102, 224-233.

Zaman, B., y Mifsud, C.L. (2017). Editorial: Young children's use of digital media and parental mediation. Cyberpsychology: Journal of Psychosocial Research on Cyberspace, 11(3). http:// dx.doi.org/10.5817/CP2017-3-xx 